\title{
IntuModels: Enabling Interactive Modeling for the Novice through Idea Generation and Selection
}

\author{
Yen-Ting Cho \\ choyenting@ncku.edu.tw \\ National Cheng Kung University \\ Taiwan
}

\author{
Yen-Ling Kuo* \\ ylkuo@mit.edu \\ Massachusetts Institute of Technology \\ United States of America
}

\author{
Yen-Ting Yeh* \\ y6yeh@uwaterloo.ca \\ University of Waterloo \\ Canada
}

\author{
Yen-Yi Huang \\ e74036132@gs.ncku.edu.tw \\ National Cheng Kung University \\ Taiwan
}

\author{
Po-Lun Huang \\ pa6071096@gs.ncku.edu.tw \\ National Cheng Kung University \\ Taiwan
}
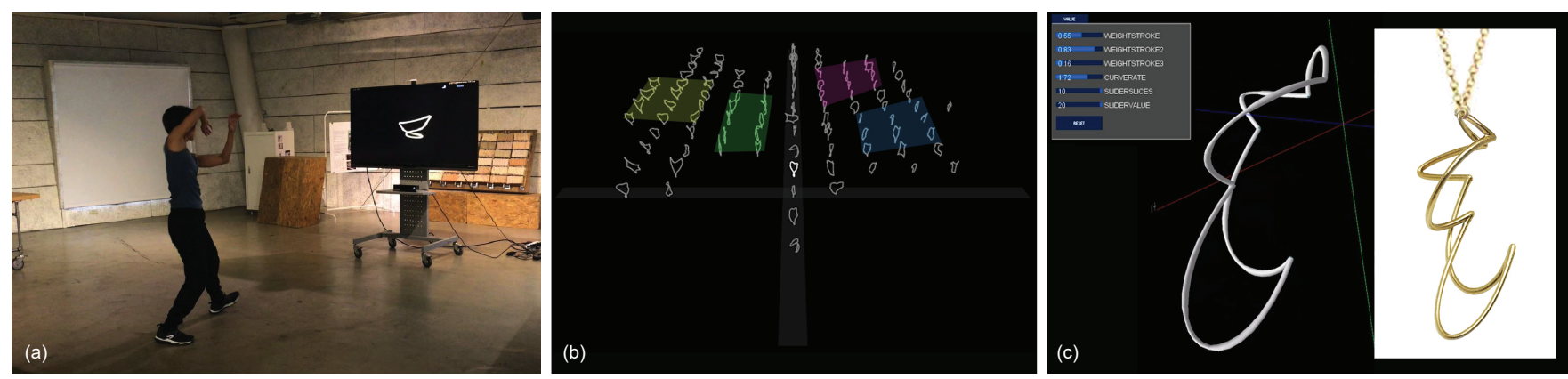

Figure 1: IntuModels workflow. (a) Idea Generation: Users can easily create models using their body movements. (b) Idea Selection: Similar model groups are presented together (the same color overlay represents similar groups) for guided exploration. (c) Refinement (left), Fabrication (right): Users can fine-tune the selected model by adjusting parameters before printing.

\section{ABSTRACT}

We present IntuModels, a machine-assisted interactive modeling workflow to enable the novice to create 3D models. The workflow uses a phase-driven approach, including idea generation and selection, to stimulate creativity and assist users who are not familiar with generating design ideas and 3D modeling techniques. By transforming parametric models using continual input data controlled by users, IntuModels motivates users to intuitively generate a huge amount of 3D model options with a good diversity. For selecting from the created models, we design a balanced overview showing the models using clustering and staged tools to help the user view and understand the models correctly. We tested IntuModels with a sample of novices who were asked to create a wire-based jewelry model. Presented in thematic networks and quantitative charts, the results showed that the novice considered IntuModels to be intuitive to use and useful for creating models that exceeded their expectations for post-production.

${ }^{*}$ Both authors contributed equally to this research.

\section{(i) (2)}

This work is licensed under a Creative Commons Attribution-Share Alike Internationa 4.0 License.

C\&C '21, fune 22-23, 2021, Virtual Event, Italy (C) 2021 Copyright held by the owner/author(s) ACM ISBN 978-1-4503-8376-9/21/06.

https://doi.org/10.1145/3450741.3465241

\section{CCS CONCEPTS}

- Human-centered computing $\rightarrow$ Interactive systems and tools; Empirical studies in interaction design.

\section{KEYWORDS}

creative support; 3D modeling; personalization; design brainstorming; design idea selection.

\section{ACM Reference Format:}

Yen-Ting Cho, Yen-Ling Kuo, Yen-Ting Yeh, Yen-Yi Huang, and Po-Lun Huang. 2021. IntuModels: Enabling Interactive Modeling for the Novice through Idea Generation and Selection. In Creativity and Cognition (C\&C '21), fune 2223, 2021, Virtual Event, Italy. ACM, New York, NY, USA, 10 pages. https: //doi.org/10.1145/3450741.3465241

\section{INTRODUCTION}

Digital fabrication enables people to quickly create physical models and prototypes. In order to create a model, current fabrication processes require sufficient knowledge of computer-aided design (CAD) tools and geometric representations. While previous projects have explored how to make tools and computational design more accessible for people who do not have specialist knowledge [11, 12], our preliminary research underlines that novices still have questions about what to make and how to make with 3D digital modeling tools. We broadly refer to users who are interested in customization and crafting but do not have the required 3D modeling knowledge or skills as novice. The target users are novice crafters or general users who may or may not 
have prior experience in $\mathrm{CAD}$ tools. Whilst novices have the intent to make, they do not know how to make using 3D modelling.

The creation process usually involves these phases: ideation, design, prototyping, production, refinement. In this paper, we propose a new machine-assisted workflow, called IntuModels, to enable people to produce 3D models using an indirect and phase driven approach, inspired by brainstorming, so that the novice, who has no 3D modeling background, can easily create their own 3D models. The goal of IntuModels is not to design a 3D CAD tool, which is mostly used in the later prototyping and production stages. Instead, we aim to address the challenges novice makers face in the early ideation and design stages (what to create), as well as their lack of professional background in 3D modeling (how to make).

We combine multiple techniques, such as motion sensing, parametric design, and clustering, to address the challenges presented above. IntuModels is an interactive modeling workflow comprising two main phases: idea generation and idea selection to further encourage an implicit initial intention to be explored and transformed into more complex and multiple options. We demonstrate and test the IntuModels workflow with wire-based jewelry creation which allows users to create abstract and concrete shapes by moving their bodies to interact with the selected parametric models. The created model options are process-driven, not random, and can be post-produced to meet users' needs.

To overcome the two main challenges of the novice's unfamiliarity of 3D modeling and generating a lot of ideas, we validate IntuModels through discussions about: (1) intuitive experience, (2) idea encouraged interaction, (3) clear idea overview, and (4) staged selection process. IntuModels enables novices to brainstorm efficiently by providing an holistic overview of the model options using machine learning techniques. The information it provides and fast ideation helps users to make informed decisions and quickly reflect on the options available to them.

In summary, this paper makes the following contributions: (1) we propose a machine-assisted workflow that augments the interactive modeling process with idea generation and selection, (2) we identify that combining parametric models and designed transformation can stimulate novice users to create a large number of diverse 3D models, and (3) we demonstrate an intuitive interface where novice users can create a 3D model without any prior experience.

\section{RELATED WORK}

\subsection{Interactive Modeling for Digital Fabrication}

Designers and modelers use CAD software to create and view 3D fabricable models. In addition to general-purpose CAD software, several tools are developed for specific shapes or forms [21, 29, 37] However, most of them are developed for professional design practice and, consequently, novices find them too complex or difficult to learn for their needs.

Some existing projects, e.g. Spatial Sketch [32] and the Sketch Furniture project [7] directly use human body movements to make model creation more intuitive. However, no design parametric transformation is applied to the output form/model controlled by body movements. Some novices might lack the confidence to create 3D models using their body movement because the output models may not be deemed to be good if they do not move in the 'correct' way.
Interactive fabrication [22,33] is a recent attempt to enable users to directly manipulate 3D models in a physical fabrication workspace rather than in a digital editor. For example, ShapeMe [31] and FormFab [23] captures the cuts and finger movements made by the designers/makers to change the $3 \mathrm{D}$ models in the workspace. These allow users who are not familiar with CAD tools to edit models in place. However, novices who are new to 3D modeling may not have ideas about what to make or change even when using these intuitive tools. We design and transform parametric models and incorporate an idea generation phase to convert users' body movements to 3D models. This makes novices feel less concerned about their skill level and enables to easily create a large amount of 3D models.

\subsection{Design Idea Generation}

Both experts and novices seek design ideas for digital fabrication. Many existing digital fabrication tools [30] allow users to select modules or parts from a library to create a fabricable object. Existing objects are often included in 3D creativity tools as typical consumers may want to 3D print objects to repair an existing item(s) [1, 27]. While libraries are familiar metaphors to many users, they might not necessarily be user-friendly to novices, who may not be able to identify which item in the library they want to apply.

Brainstorming is one of the most used ideation processes to generate design ideas. It promotes creativity by encouraging and generating a huge quantity of ideas while deferring judgments to later stages in the process [25]. Several tools have been developed to use cards [4, 9], tangible objects [5], and LEGO [15] to inspire participants to generate more original ideas in a team-based setting. IntuModels focuses on design idea generation in single-user settings; and instead of directly supporting brainstorming, it builds on the ideas from brainstorming to enable the novices to create $3 \mathrm{D}$ models in a comfortable setting.

\subsection{Design Space Exploration}

In order to identify a good design solution, users need to explore the whole design space strategically. Graphs and network structures are common tools to connect different design choices for users to explore by following the edges of the network [3, 17, 35]. However, novices may not know how to navigate the network structures since they are not familiar with typical design practice. Outside of network structures, projecting multi-dimensional data onto a $2 \mathrm{D}$ space $[14,28]$ or using sliders [16] are also used to enable users to parallel explore different aspects of the designs. We take a similar approach to map 3D models to a 2D space. Presenting all possible models in a 2D space allows novices to navigate and compare the models that interest them.

Since the idea generation phase may create a large volume of design options, the number of possible 3D models usually exceeds the amount a typical user can handle. Dream Lens [20], for example, explores models by summarizing designs into multi-attributable grids and stacking multiple similar models onto one composite 3D view so that users can see the attributed relationships and compare models. Whilst such tools are powerful for designers to explore and identify the differences between models, novices tend not to understand the meaning of all attributes, or how to compare 3D models. Rather than showing the relationship between attributes, we use hierarchical clustering to reveal meaningful relationships between the user-generated 3D designs. 
Some digital fabrication or folded UI creation tools $[26,30]$ also provide machine-assisted selection phase to help users narrow down to a $3 \mathrm{D}$ model they like. To make the exploration and selection easier for novices, we adopt a staged interface to reveal the details/information cumulatively.

\section{DESIGN RATIONALE}

Designing an interactive modelling software for novice makers to use in the early creation process needs to address two core issues the users face: (1) what to create and (2) how to make. Inspired by brainstorming, especially the principle of deferring judgement [ 24 34], instead of asking users to create the intended models, we design the workflow to encourage users to easily 'explore' the design space of 3D models to create a huge number of options first and then select the best one from the options. This leads to the two major phases of our workflow: idea generation and idea selection. These two phases not only allow novices to see the models first to get design inspiration, but it also eases the stress of making in 3D environments. In this section and throughout this paper, we aim to discuss this central research question: How can a machine assisted workflow and a phase driven approach inspired by brainstorming enable interactive modeling for the novice? We also discuss the principles that are useful in different phases.

\subsection{Idea Generation Phase}

What to create - In this phase, we have to encourage users to explore more diverse model options in order to discover the one they like. Cross-fertilization is one way to combine, synthesize, and stretch ideas to get more diverse ideas in a typical brainstorming process [34] Taking this principal, we can enable users to generate more diverse 3D models by designing an intuitive non-linear relationship between input modalities and output models. We refer the mapping between user input and the generated model as design transformation. A good transformation ensures that any input can introduce enough variations in the output 3D models which also encourage users to explore beyond their expectation.

How to create - As discussed in 2.1, existing 3D modeling software are not easy to use for novices as they will generally only have enough knowledge to operate the software to manipulate 3D models after going through a steep learning curve. Consequently, novices need some assistance to know what to do. However, we avoid produce a 'step-by-step' guidance as it limits creativity, instead, we suggest that parametric models serve a great middle ground. A parametric model defines the design space as a set of parameters for users to change. It turns free-form interactions into a predefined boundary while maintaining the diversity of the models. Defining the parameters and the relationships between parameters are crucial to enhance the output diversity. We need to ensure the dynamism and flexibility of the relationships of parameters to reflect the detailed changes of the given continual input data, e.g. body movements, as they are intuitive ways for users to interact with the versatile model and provide enough input changes to generate a large number of output models.

\subsection{Idea Selection Phase}

What to create - After users generate a huge number of 3D models by exploring the parametric design space, a balanced overview of the created models is essential to help them navigate through the models and explore the variations for selection without feeling overwhelmed by the vast data. Machine learning methods such as clustering and projecting in vector space are useful to organize the data in terms of the attributes we care about for 3D models. We can utilize these techniques to reduce the number of models presented to the users while allowing them to compare the differences between models.

How to create - 3D interfaces can be very confusing for nonexpert users [13], including understanding the model correctly on a 2D screen, navigating spatially though a world full of 3D models, and becoming familiar with the parameters for modeling. In order to help them to understand the models given their 3D skill level, our selection phase should reveal necessary 3D information gradually and allow them to easily review the models from multiple perspectives. To achieve this, we use a staged process to guide users to review the models at high level of granularity and provide them with various tools to enable them to build a correct understanding of the models through stages.

\section{INTUMODELS}

IntuModels combines idea generation and idea selection in a unified design process to enable novices to create 3D models. IntuModels first asks users to generate a large amount of 3D models using body movements and then guides them with an interface to explore and refine the generated designs. By splitting model generation and selection into two separate phases, novices do not need to be concerned about creating the 'best' model in the generation phases. In the following, we use wire-based jewelry design as an example to show how IntuModels works in practise.

\subsection{Idea Generation}

To enable novice users to generate a large number of different 3D models, the generation process needs to be intuitive, motivate them to be creative and allow them to make changes during the process.

Body Interactions and Continual Input - IntuModels sets up a depth camera to track users' body movements and translates movements to 3D parametric models as body parts are intuitive controls [32] for users who are not familiar with 3D modeling. See Fig 1 (a) for the setup. The shape of the 3D models is controlled by the users with their body movement. The user can observe the real-time and continuous changes in the output models to figure out where to move next. By recording the changes with a reasonable frame rate, IntuModels can generate a large amount of 3D models with fine movements. The input body movements can be replaced with other modalities, e.g. facial expression, as long as they provide variety and are easy to use for novices.

Parametric Model - In IntuModels, we focus on the aesthetic aspect of the 3D models rather than the functional aspect of the fabricated objects. The output forms are determined by the designed parametric model and the input body joints. To keep the process simple, we use five cubic Bézier curves as the 3D wire-based jewelry model. The control points of the Bézier curves are the weighted sum of the input 
body joints. The weight of the joints determines the curvature of the wires. The choice of model components and parameters set the aesthetics of the output 3D models, whereas the input body joints create the variety of the outputs. Not limited to parametric curves, the design of the parametric models can incorporate other forms such as surfaces and polygons to expand the possible output set. (Fig 2)

Design Transformation - Designing the transformation not only defines the space of the output 3D models; different types of transformation from body movements to parametric models can motivate users to make changes to the model in different levels. Other than direct one-to-one mapping between the input joints to the curve control points (see Fig 3 (a)), an indirect design transformation may use multiple input joints and other information, e.g. speed or acceleration, to change the curve control points (see Fig 3 (b)). This indirect transformation can introduce a level of unexpectedness to users. Users need to move their body more to explore the possibility of the output forms and figure out the relationship between their movements and the 3D models. This indirect transformation also reduces any stress caused by novices' using unfamiliar tools to create what they perceive to be a 'good' result. In particular, we adopt two transformations in IntuModels to create forms shown in Fig. 3 (b). (1) The location of a skeleton point is used multiple times. A skeleton point is not only used as an end point but is also used for weighing the calculation of the control points of two of the adjacent curves next to the connecting curves. For example, the left hand and right feet (orange dots) affect the control points of the curve between the head and right hand. Whenever a user moves one body joint, four nearby curves are updated. (2) The accelerations of the skeleton points are used when calculating the adjacent control points. For example, we use the acceleration of the head movement to compute the weight of the head when calculating the control point from the skull and the skeleton of the left hand and right hand. Both transformations create the indirect mapping between body movements and the output models.

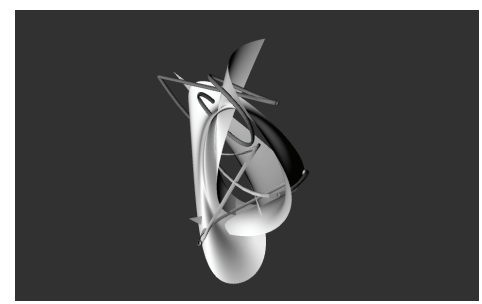

Figure 2: Parametric model made of Nurbs surface and spline curves.
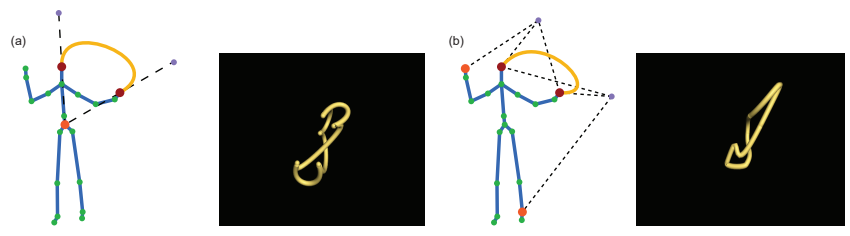

Figure 3: The formation and the rendering of (a) the NONTRANSFORMED MODEL, and (b) the TRANSFORMED MODEL

\subsection{Idea Selection}

Novices need more guidance to explore the large volume of generated models. In order to provide meaningful guidance to the users, IntuModels applies machine learning techniques such as clustering and dimensionality reduction to organize the 3D models to make them easier for users to understand and compare. We also introduce a staged selection interface to enable novices to view and select ideas with high level of granularity.

4.2.1 Understanding the Generated Ideas. After generating a large number of models, it is hard for users to browse through all models to select the ones they like. To reduce the information overload while keeping the diversity of the generated designs, we group similar designs together and present them in a $2 \mathrm{D}$ space to summarize the generated ideas.

Grouping Similar Design Ideas - The goal of grouping is twofold: (1) It reduces the number of models for users to review in the idea selection phase as users cannot compare all models to see the detailed differences between them; (2) It serves as a way to quantify the diversity of the generated ideas; users can easily see the landscape of 3D models. We use a clustering algorithm to group models into a manageable size of groups for users. The input features for the clustering algorithm are the parameters of the 3D parametric models. In IntuModels, the parameters are the users' skeleton positions modulated by damping and acceleration. We choose affinity propagation [6] to cluster models because it is a non-parametric method. This is important since different users create models of different varieties. The target number of clusters is hard to estimate $a$ priori. After clustering, each group may still contain too many models, which makes it difficult to compare models. We therefore need to perform a second clustering inside the groups which contain too many models, i.e. 20 in our implementation. This hierarchical clustering not only maintains a manageable size for each cluster, but also provides a way to compare models at different levels.

Projecting Models onto a 2D Plane - To display these clusters, we choose the model that is the center of a cluster to be the representative model of that cluster. We aim to arrange cluster centers on a $2 \mathrm{D}$ plane because novices are generally more familiar with 2D. We use $t-$ SNE [18] to project the models from their high dimensional parameter space onto 2D space. t-SNE tends to arrange similar data points close together. Therefore, similar clusters are closer in this 2D plane. It is possible to use shape similarity measures such as Fréchet distance to arrange models in 2D. However, they are usually shape dependent. In order to accommodate different parametric models, i.e. models other than curves, we chose dimensionality reduction based on raw features. Fig. 1 (b) shows the projection of cluster centers on a 2D plane; the different colored overlays indicate the proximity of similar model groups.

4.2.2 Staged Selection Interface. IntuModels presents a staged selection interface based on the clustered groups to guide users to select a 3D model to digitally print. Users first see an overview of the clustered models. They can navigate the landscape to see the 360-degree view of the representative model for each cluster. They can then go into the cluster they are interested in to compare similar models in some detail. Having identified the final model, users can refine it by adjusting the parameters that control the shape of 3D models. At each 

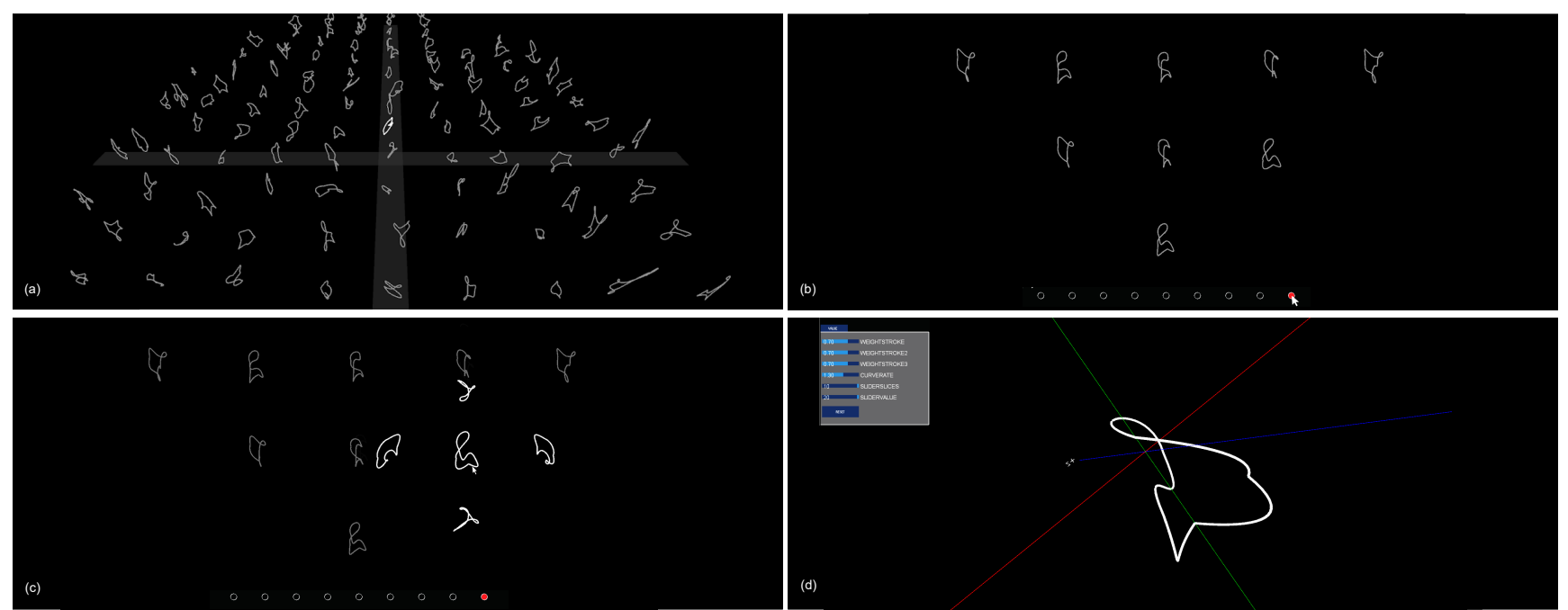

Figure 4: Idea selection interface. (a) Overview of the main model groups; (b) Detailed comparison and variant selection; (c) Four different views around the hovered model; (d) Refinement.

stage, we reveal different information to users by providing various tools that show different perspectives of the model.

Stage 1: Overview of Model Groups - In the stage 1, we show the representative models from the first clustering result. We call these clusters 'main model groups'; see Fig. 4 (a). All the main model groups are presented to users at once to show the full landscape of the generated models. In order to help users search through the model groups, we take the $2 \mathrm{D}$ projection produced by t-SNE, descretize the positions, and arrange the models in a 2D grid. This grouping of models helps users to identify rules and the relationship between models. The grid system also makes it easier for users to navigate. This is also useful for users who are not familiar with 3D environments so that they can start viewing the model from a $2.5 \mathrm{D}$ presentation instead of getting overwhelmed by the 3D information.

Users can change the highlighted model and further zoom in and out to compare models group-by-group first, then model-by-model. Once a model is 'focused', or highlighted, users can click the autorotate button to rotate a model automatically to see the model from other viewpoints. This feature enables users who are not familiar with controlling a model in 3D to see all perspectives of a model without being distracted by having to control the angle. If a user gets lost in the 3D environment, they can double-click on the screen to reset the environment.

Stage 2: Detailed Comparison and Variant Selection - Users select a main model group from stage 1 to explore its subgroups. Stage 2 shows different subgroups in different pages so that users can compare details within each subgroup before choosing a model; see Fig. 4 (b). The representative model of the current subgroup is in the middle of the top row. Other models in the same group are sorted by the similarity to the representative model and arranged around the representative model from the left to the right. At this stage, the models in each subgroup can be very similar to each other. Consequently, we designed an instant 4-angled view; see Fig. 4 (c). When users hover over a model, views from four different angles pop up around the models so that they can understand the models correctly. Users can simply compare two models by hovering over models in sequence.

Stage 3: Refinement - Before outputting the selected model for post-processing and 3D printing, we enable users to fine-tune their selected model in a full 3D setting; see Fig. 4 (d). The skeleton points of the selected model is locked in this stage. Users can adjust other parameters such as line weight and curve rate using the top-left panel and rotate freely to examine the final output. If they are not satisfied with the resulting model, they can always reset the parameters or go back to previous stages to select a new one. We designed the staged experience to help users understand their model correctly, adding more and more information stage-by-stage, so they will not be overwhelmed by the volume of models and 3D information.

\section{EXPERIMENT 1 - DESIGN TRANSFORMATIONS}

To understand the design transformations, i.e. the way to map body movements to 3D models, encourage diversity of the generated models, we tested the proposed TRANSFORMED MODEL against the NONTRANSFORMED MODEL (Fig. 3). The NON-TRANSFORMED MODEL also consists of five cubic Bézier curves. However, the control points are the simple extension from spine base to the two end points, which means the curves are the direct mapping of the body movements.

\subsection{Setup}

5.1.1 Participants. We recruited 5 participants (2 males, 3 females, aged 20-40) with no prior experience of 3D modeling.

5.1.2 Apparatus. We used Microsoft Kinect One to capture full body movement data and store the positions of 25 skeleton joints at each time frame. We applied a springing effect to the captured skeleton positions to produce a smoother animation of body movement and higher model generation rate. Our final system frame rate was about $40 \mathrm{fps}$, which is higher than the frame rate of body tracking ( $30 \mathrm{fps}$ ) and leads to a large amount of generated models with smoother changes between models at adjacent timestamp. Participants were 
asked to stand two meters in front of the Kinect and the display screen and then move in a $2 \times 2$ meter space. This setting ensures better tracking resolution.

5.1.3 Procedure. The participants were asked to create their own model based on their full body movements. They were instructed to consider their body posture, movement patterns, and speed of movement whilst moving their body. No specific target output was given and the results could be either abstract or concrete shapes. Participants were asked to understand the relationship between movement and the forms of output models, and create their favorite models during the practice and experiment sessions. They were also told to explore similar body movements once they identified the models they were most interested in to manipulate and further develop the desired model(s). The model generation phase was divided in two parts: creating with the NON-TRANSFORMED MODEL and the TRANSFORMED MODEL. Each part began with a 2-minute practice session. Participants could freely move in front of the camera and display to get familiar with the 3D forms. A 30-second recording session was conducted after the practice. Each recording generates 1000-1300 3D models. The order of testing models was interleaved. After the generation step, participants explored the created forms and selected the favorite one. At the end of the experiment, we conducted a semi-structured interview to elicit subjective preferences and general feedback. The experiment lasted about 40 minutes on average.

\subsection{Results}

5.2.1 Semi-structure Interview. The semi-structure interview ${ }^{1}$ consisted of the following three parts:

- Interactive modeling: This part evaluated the diversity of the generated models and how it can stimulate the creation process 3 participants said that the 3D models generated using the TRANSFORMED MODEL show greater diversity. All participants agreed that the TRANSFORMED MODEL could stimulate their motivation to move their body further to see more distinct models.

- Output: This part evaluated the expectation of outputs. 3 participants thought the 3D models generated using the TRANS FORMED MODEL looked aesthetically better than those using the NON-TRANSFORMED MODEL, while 1 participant felt that there was no difference between the two models. 4 participants said they could create 3D models that exceeded their expectation using both models; the other participant felt that the generated 3D models were unexpected when using the TRANSFORMED MODEL.

- Overall experience: This part evaluated the participants' experience of the whole workflow. 3 participants agreed that the TRANSFORMED MODEL could help produce more distinct and unexpected models.

5.2.2 Observation. We summarize participants' feedback as:

- Number of models. 3 participants were surprised by the number of models they created in 30 seconds. "The number of models is surprisingly large for 30 seconds." (P5)

${ }^{1}$ Full interview questions and additional graphs of thematic networks are provided as supplementary materials.
- Easy to design. One participant pointed out that they could easily create models that looked professionally-designed using simple movements: "I feel like every result can be sold and related to myself." (P2)

- Greater diversity that stimulates creation. All 5 participants reported that the whole procedure can create diverse models and stimulate their creativity. P5 commented that, "when interacting with the TRANSFORMED MODEL, I need to have more and larger movements to understand the model rules ... so this encourages me to think about what movements can form the model I want to see." P3 said that "the TRANSFORMED MODEL produces more kinetic energy when I move my body and creates more unexpected results. This stimulates me to imagine the next result while performing different movements iteratively."

- Confidence. Transformation creates clear responses, which enhances participants' understanding of the interaction: "The transformed model gives me stronger responses; I can see the relationship between my body and my models." (P3) Furthermore, the parametric transformation is created by participants' body movement, therefore each movement creates a different transformation and shows related options based on the current body movement: "Transformed models give a larger and moving output form, so I can see the results created by my intended movement, which encouraged me to try more." (P2)

5.2.3 Implications. The diversity of the generated models is greatly enhanced by design transformation. This diversity reflects both the quantity of the original ideas (i.e. the distinct models) and the complexity of the produced models. This can encourage users to explore the possibilities of the output forms and express themselves. The transformed models also enrich the user experience during idea generation phase. Because of their uncertainty about the transformed models, users are motivated to repeat their movements and make iterative changes to understand the transformation.

\section{EXPERIMENT 2 - INTUMODELS WORKFLOW}

We tested the IntuModels workflow with jewelry design sessions to investigate user satisfaction with the final output, the usability of the staged selection interface, and the overall creation experience. We chose jewelry as the experiment output because it is an easy medium for the novice user to create; jewelry is a form that everyone is familiar with and can be designed in many styles [10]. Users can create jewelry for multiple purposes, e.g. for personal use, to gift, etc. We can collect data about their intention to create and personal experience of creating during the experiments, and focus on discussion of idea brainstorming and selection to create a process-driven creativity support environment. We evaluate IntuModels without comparing against a baseline system because we focus on understanding the usability, effectiveness and impact of our design procedure for novices, who are an underserved user group for most existing modeling systems.

We recruited 14 participants (6 males, 8 females, aged 20-50) for our design session. All participants were motivated to create their own creative and customized jewelry output. All had very limited or no prior experience of using digital modeling software. We used the same setting as Experiment 1 with an improved system frame rate at 

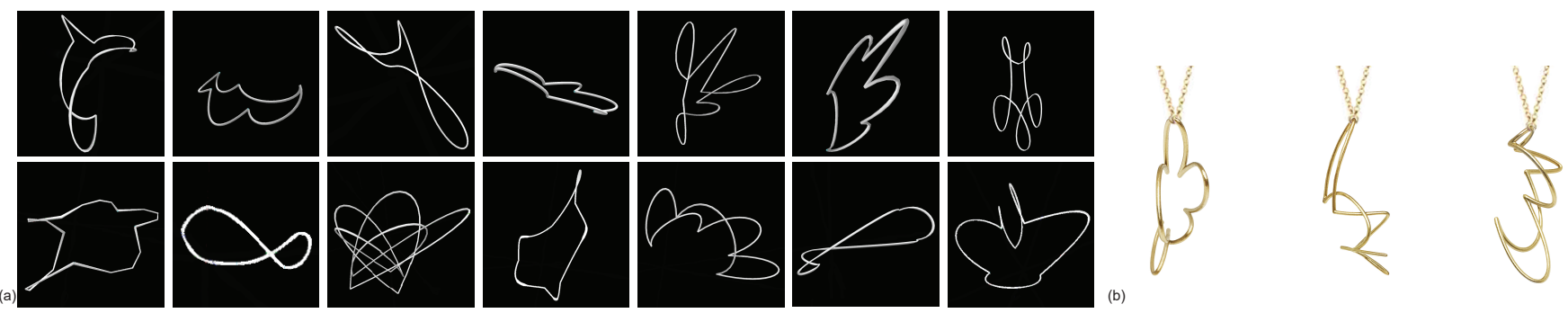

Figure 5: (a) The final 3D models created by all 14 participants using IntuModels. (b) Selected 3D printed participants' creation.

50-80 fps in order to generate more models. In a 30-second idea generation session, 1500-2500 3D models were generated and recorded. The experiment procedure was the same as Experiment 1, with only the TRANSFORMED MODEL was used. A post-session interview was conducted after the design workflow. The whole experiment lasted about one hour on average.

\subsection{Evaluation Methods}

We integrate both quantitative and qualitative data collected from the post-session interview to evaluate IntuModels. A form of keyword sorting and thematic network $[2,19]$ was used to identify themes and organize the information into a network diagram to capture the intricacies of meaning within the rich textual data from questionnaires [8]. We also collected usage logs from all experiment sessions to reveal data showing participants' operation through the experiment. We triangulated the quantitative data and qualitative data to draw conclusions [8].

We conducted a semi-structure interview with each participant at the end of the design sessions. Participants were asked to give subjective ratings with a 9-point Hedonic scale (higher score is better) and open-ended feedback. The questions ${ }^{1}$ can be grouped in the following categories:

\section{- Overall}

- Satisfaction of the final output: How satisfied is the user with the output model after creating with IntuModels?

- Uniqueness of the final output: What are the differences between this creation and other jewelry the user has seen?

- Idea generation

- Body usage: The user describes their experience in using body movements to create models.

- Awareness of clustering arrangement : Is the user aware that similar model groups are closer to each other?

- Staged selection interface

- Usability: The usability of the selection interface. Does the interface help the user make jewelry?

- Usability of clustering arrangement: Does the clustering arrangement help the user choose models?

- Search/Navigation: How complex is the layout for the user to search models?

- Learnability: How hard is it to learn to navigate in the overview?

- Expectation: Is the form of 3D model as predicted when the user enters the refinement stage?

- Features: Which tools in the interface are useful for the user?

\subsection{Results}

While participants all started from the same parametric model design, the forms and designs were very different between participants given their different movements. (Fig. 5)

6.2.1 Quantitative Data. We show the percentage of users who 'greater than very agree' (i.e. very agree, strongly agree, and extremely agree) with the statement as well as the score distribution in Fig. 6 (a). 13 participants (93\%) were satisfied with the created models. All participants thought the selection interface was useful and easy to learn. 11 participants (79\%) found the interface layouts are easy for them to search models. 12 participants $(86 \%)$ noticed the form-related arrangement, and 10 participants $(71 \%)$ found this arrangement useful for them in the idea selection stage. The results show that the participants were not only aware of the clustering arrangements in the interface, but also acknowledged its usability.

Fig. 6 (b) ranks the tools in the selection interface rated useful by the participants. Tools that provide new perspectives of the models, e.g. 3D interface in the refinement stage, zoom in/out and auto rotate the selected model in the overview stage, and the four-angle views of models in the detailed comparison stage, are the most useful ones for the participants. These tools allow users to compare different views to see the differences between models. 11 participants also found it useful to reset the model angle with one click when they got lost in rotating the model. Overall, 13 participants (93\%) said that the models they saw in the refinement stage were as they predicted because they had enough information to build a good 3D understanding of the selected model.

6.2.2 Qualitative Data. Each of the remarks can relate to more than one cluster and we use thematic networks to display the organization of the verbatim remarks $[2,19]^{1}$. For idea generation process, four organizing themes emerged: (1) intuitive experience, (2) large amount of models, (3) exploratory interaction, and (4) unexpected models. We grouped the four organizing themes to form a higher order premise: IntuModels enables and motivates the novices to intuitively create a lot of model options with a good variety and without worrying about their lack of modeling experience.

For the idea selection process, five organizing themes emerged: (1) easy to navigate, (2) compare easily at stage 1 , (3) compare easily at stage 2, (4) clear overview, and (5) holistic information. We grouped the five organizing themes to form a higher order premise: the staged selection experience is easy to navigate, and actively helps participants to gain a holistic understanding of the models with the provided tools to make a desired final selection. 

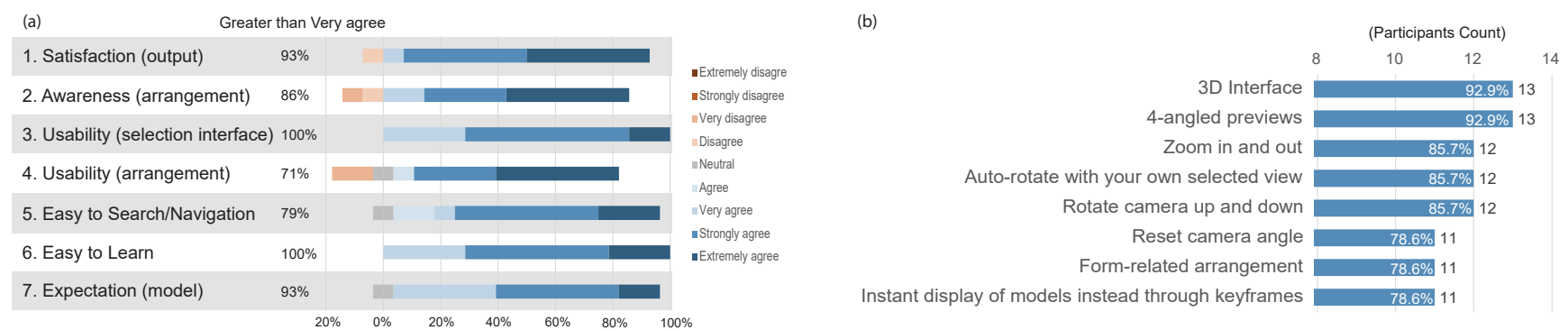

Figure 6: The quantitative results. (a) The percentage of agreement and the score distribution of the ordinal questions in the follow-up interview. (b) Number of users rated a tool useful in the selection interface.

The summary of textual data helps us understand each participants' experience. In the following part, we further synthesize the findings with the data captured from other methods for a holistic discussion.

\section{DISCUSSION}

\subsection{Idea Generation: Intuitive Experience}

The keywords show that 10 out of 14 participants said that IntuModels is easy to use. P12 said: "IntuModel helps me to create jewelry without modeling experience." P1 from the experiment 1 stated: "Very easy to use. I can simply create results using my body movement." IntuModels is easy to use and it enables each participant to create an average 2400 models in 30 seconds. P14 said that "The selection interface shows me that the contribution of my action is abundant." $\mathrm{P} 4$ also mentioned that "The quantities of models generated are massive."

Participants noted that using IntuModels is not complicated or stressful. For P3: "I can create models without worrying about how to draw.... I can also create freely without thinking about the limits of physical tool for modeling." P7 said that IntuModels "helps me create unconsciously (no stress)." P12 stated: "I created the jewelry using IntuModels but hardly noticing the modeling process." Participants can, then, create many model options without worrying about their lack of modeling experience.

\subsection{Idea Generation: Idea Encouraged Interaction}

Regarding the indirect interaction and transformed models, P6 said that "I need to reconnect my body and mind, the unfamiliarity required me to explore for longer". Participants also noted that the unexpected projected models encouraged them to explore further. P5 said that "The interaction is very interesting and easy. I can explore the relation between movements and forms." Participant 10 stated "I like the curves, and [the model] accidently turned into a crown... and opened like a flower. That surprised me and made me think outside of the box."

At idea selection stage 1, many participants noted that they saw the unexpected models created by themselves. 11 out of 14 participants stated that IntuModels gave them "abundant" and "unexpected" options. P6 said that "there are many surprising models at stage 1 for me to choose from." P4 stated: "Everyone moves differently. The model is flexible enough, so the look of the result is abundant." Participants noticed they had created a lot of different model options after the interaction and after the models were presented in the interface.
9 out 14 participants stated that IntuModels encouraged exploration of model options. We thus conclude that the transformed parametric model created unfamiliarity and unexpected results which motivates novice users to explore further. As a result, they create more model options, in terms of both quantity and variety.

\subsection{Idea Selection: Clear Overview}

$86 \%$ participants acknowledged the positive effect of clustering. Furthermore, if we review their comments, 12 out of 14 participants said that they could see the model grouping clearly. 11 participants agreed that the grouping was very helpful when searching for their ideal main group. 10 participants mentioned that it was useful to see all main groups in stage 1 at the same time. P1 said that the stage 1 looked like a "virtual showroom." P7 said that grouping helped them to "see the rhythm of the main types of the models and sequence of body movements. It is like an archaeological site." P13 stated that they could "search systematically and strategically."

$71 \%$ participants said that clustering is very helpful to choose the desired model. Just 2 (14\%) participants felt that the grouping was not very useful because their main groups all looked different from each other and, for them, the groupings were not obvious visually.

We conclude that a good overview of models helps participants to see the unexpected and unique results and to quickly narrow down options for a final model.

\subsection{Idea Selection: Staged Process}

At stage 1, 12 out of 14 participants agreed that providing 3D information together with the zoom-in and zoom-out tool from the first stage was useful. 12 participants agreed that it was useful that they were allowed to reset the camera angle. We know from previous research that a 3D interface can be very confusing for the novice user [13].

13 of the 14 participants agreed that the four angle views at stage 2 was useful because the feature helped them compare similar models in the sub-group in detail. With all the extra 3D information provided in stages 1 and 2, when entering stage 3, we asked participants how much the model matched their expectation, and $93 \%$ of participants agreed that the model was what they expected. The usage log also shows that the average back stage key press number is only 3.14 . Participants do not need to go back to the previous stage often to reselect their models.

93\% participants agreed that they were satisfied with the final results. P12 said that, "through stages, more and more 3D information has been revealed gradually", so they were not overwhelmed by 


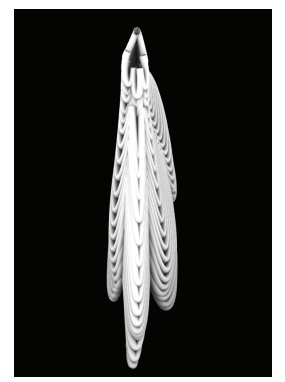

Figure 7: An example of models created using participant 3's sequenced data.

the number of models and quantity of 3D information. In summary, staged experience reduces the stress of selection, also actively helps participants to gain a holistic understanding of the models with the provided tools and enables them to select a final model.

\subsection{Limitations}

Participants who do not move their body freely might not be able to create enough model variety. If so, parametric transformation is useful for suggesting the alternative forms for brainstorming while still maintaining a close relationship between the output and participants' body movement. Also, when participants' main groups look very different from each other, the projected layout in stage 1 does not help users compare the visual similarities between models. Reminding participants to repeatedly explore their form during interaction can increase the visibility of their favorite main groups.

Some participants mentioned that they like the abundant unexpected results, but it will be helpful to them to know which body movements are used to create these models; revealing this link will strengthen the personal experience and highlight the meaning and uniqueness of the outputs. In order to keep the simplicity of the selection stage, we suggest adding this information as a supplementary feature.

\subsection{Applications}

The goal of IntuModels is to help participants explore and select from a huge amount of data swiftly and intuitively. Participants use the form to prototype their physical craft creations. They can also input the selected model into a CAD tool for further refinement and exploration of the related models by simply searching around the selected model's time point as the body movement is continual. In other words, IntuModels applies a spatial interface to present the created sequence of models and users can take advantage of the temporal metadata of the models and explore them sequentially in CAD software. This is useful for studying the macro form movement as well as sequencing the forms to create a larger object [36] (See Fig 7).

\section{CONCLUSION}

By incorporating a design ideation process, using parametric design and design transformations, novices were able to easily create a large amount of 3D models. Experiment results suggest that we achieved a good balance between intuitive design and surprising the user with unexpected results. For idea selection phase, the accumulative and holistic understanding of the models actively helps novice users to comfortably select a final model for further production.

We focus on novice and crafters, but IntuModels can benefit:

- Interactive modeling tools: IntuModels provides design recommendations for future CAD tools for novices.

(1) Design transformation helps novices generate designs which are beyond their original intention. By applying design transformation in CAD tools, e.g. multiple parameters are affected when changing one parameter, novices might be motivated to generate diverse and surprising results.

(2) If there are many model options, the staged selection interface with clustering can be used to improve the selection interface in CAD tools. This machine assisted method is adopted and adapted in future projects to effectively reduce design options, such as generative design for all users. The efficacy of the overview design in the interface stage $1 \mathrm{can}$ be modified by considering extra parameters (e.g. distance between groups) or alternative layouts for an even more complex selection task, or for presenting clearly a set of models that are very similar or different.

- Customization platform: Most customization interfaces provide a linear experience for users to select the design of each part. The phase-driven workflow of IntuModels serves as a useful reference for platforms that want to create an experience design by engaging users through intuitive option exploration and machine-assisted decision making.

- Creativity support tools: Every idea counts and should be preserved for model selection. In our workflow, every body movement is an idea and users can review and select what they like through the staged selection process. Every design iteration of a model, eg. refining the curve or scaling the box, should be preserved and then organized for users to understand effectively and reflect holistically in order select the most preferred options.

IntuModels also serves as an example of integrating multiple techniques through connecting design and HCI. We anticipate that this project will stimulate more creative methods to help the novice generate and select modeling ideas. We therefore hope that our project contributes to future research in brainstorming, digital process design and idea generation and selection in 3D modeling fields.

\section{ACKNOWLEDGMENTS}

This work was made possible by the Ministry of Science and Technology, Taiwan (MOST 108-2221-E-006-062-MY2).

\section{REFERENCES}

[1] Daniel Ashbrook, Shitao Stan Guo, and Alan Lambie. 2016. Towards augmented fabrication: Combining fabricated and existing objects. In Proceedings of the $2016 \mathrm{CHI}$ Conference Extended Abstracts on Human Factors in Computing Systems. 1510-1518.

[2] Jennifer Attride-Stirling. 2001. Thematic networks: an analytic tool for qualitative research. Qualitative Research 1, 3 (2001), 385-405.

[3] Fan Bao, Dong-Ming Yan, Niloy J Mitra, and Peter Wonka. 2013. Generating and exploring good building layouts. ACM Transactions on Graphics (TOG) 32, 4 (2013), 122.

[4] David W Chung and Rung-Huei Liang. 2015. Interaction Tarot: A card-based design of knowledge construction for brainstorming in HCI. In Proceedings of the 6th IASDR Conference on Design Research (IASDR'15).

[5] Virginia Cruz and Nicolas Gaudron. 2010. Open-ended objects: a tool for brainstorming. In Proceedings of the 8th ACM conference on Designing Interactive Systems. $85-88$. 
[6] Brendan J. Frey and Delbert Dueck. 2007. Clustering by passing messages between data points. Science 315, 5814 (2007), 972-976.

[7] frontdesign.se. 2021. Sketch Furniture Performance Design. http://www.frontdesign se/sketch-furniture-performance-design-project.

[8] Greg Guest, Kathleen M MacQueen, and Emily E Namey. 2011. Applied Thematic Analysis. Sage Publications.

[9] Eva Hornecker. 2010. Creative idea exploration within the structure of a guiding framework: the card brainstorming game. In Proceedings of the Fourth International Conference on Tangible, Embedded, and Embodied Interaction. 101-108.

[10] Emmanuel Iarussi, Wilmot Li, and Adrien Bousseau. 2015. WrapIt: Computerassisted crafting of wire wrapped jewelry. ACM Trans. Graph. 34, 6, Article 221 (Oct. 2015), 8 pages.

[11] Jennifer Jacobs and Leah Buechley. 2013. Codeable objects: computational de sign and digital fabrication for novice programmers. In Proceedings of the SIGCHI Conference on Human Factors in Computing Systems. ACM, 1589-1598.

[12] Michael D. Jones, Kevin Seppi, and Dan R. Olsen. 2016. What you sculpt is what you get: modeling physical interactive devices with clay and 3D printed widgets. In Proceedings of the 2016 CHI Conference on Human Factors in Computing Systems. ACM, 876-886

[13] Rubaiat Habib Kazi, Tovi Grossman, Hyunmin Cheong, Ali Hashemi, and George W Fitzmaurice. 2017. DreamSketch: Early stage 3D design explorations with sketching and generative design. In UIST, Vol. 14. 401-414.

[14] Hannah Kim, Jaegul Choo, Haesun Park, and Alex Endert. 2015. Interaxis: Steering scatterplot axes via observation-level interaction. IEEE Transactions on Visualization and Computer Graphics 22, 1 (2015), 131-140.

[15] Annemarie Lesage, Hubert-David Au-Yeung, Simon Bourdeau, Béatrice C Caron, and Pierre-Majorique Léger. 2019. Sketch or play? LEGO® stimulates divergent thinking for non-sketchers in $\mathrm{HCI}$ conceptual ideation. In Extended Abstracts of the 2019 CHI Conference on Human Factors in Computing Systems. 1-6.

[16] Aran Lunzer and Kasper Hornbæk. 2008. Subjunctive interfaces: Extending applications to support parallel setup, viewing and control of alternative scenarios. ACM Transactions on Computer-Human Interaction (TOCHI) 14, 4 (2008), 17.

[17] Kwan-Liu Ma. 1999. Image graphs - A novel approach to visual data exploration. In Proceedings Visualization'99 (Cat. No. 99CB37067). IEEE, 81-88.

[18] Laurens van der Maaten and Geoffrey Hinton. 2008. Visualizing data using t-SNE fournal of Machine Learning Research 9, Nov (2008), 2579-2605.

[19] Bella Martin, Bruce Hanington, and Bruce M. Hanington. 2012. Universal Methods of Design: 100 Ways to Research Complex Problems, Develop Innovative Ideas, and Design Effective Solutions. (2012).

[20] Justin Matejka, Michael Glueck, Erin Bradner, Ali Hashemi, Tovi Grossman, and George Fitzmaurice. 2018. Dream lens: Exploration and visualization of large-scale generative design datasets. In Proceedings of the 2018 CHI Conference on Human Factors in Computing Systems. ACM, 369.

[21] James McCrae, Nobuyuki Umetani, and Karan Singh. 2014. FlatFitFab: interactive modeling with planar sections. In Proceedings of the 27th Annual ACM Symposium on User Interface Software and Technology. 13-22.
[22] Stefanie Mueller, Pedro Lopes, and Patrick Baudisch. 2012. Interactive construction: Interactive fabrication of functional mechanical devices. In Proceedings of the 25th Annual ACM Symposium on User Interface Software and Technology (Cambridge, Massachusetts, USA) (UIST '12). 599-606.

[23] Stefanie Mueller, Anna Seufert, Huaishu Peng, Robert Kovacs, Kevin Reuss, François Guimbretière, and Patrick Baudisch. 2019. FormFab: continuous interactive fabrication. In Proceedings of the Thirteenth International Conference on Tangible, Embedded, and Embodied Interaction. 315-323.

[24] Alex Osborn. 2009. Unlocking Your Creative Power: How to Use Your Imagination to Brighten Life, to Get Ahead. University Press of America.

[25] Alex F Osborne. 1957. Applied imagination. New York, NY: Scribner (1957)

[26] Greg Saul, Cheng Xu, and Mark D Gross. 2010. Interactive paper devices: enduser design \& fabrication. In Proceedings of the Fourth International Conference on Tangible, Embedded, and Embodied Interaction. 205-212.

[27] Rita Shewbridge, Amy Hurst, and Shaun K Kane. 2014. Everyday making: identifying future uses for 3D printing in the home. In Proceedings of the 2014 Conference on Designing Interactive Systems. 815-824.

[28] Jerry O Talton, Daniel Gibson, Lingfeng Yang, Pat Hanrahan, and Vladlen Koltun. 2009. Exploratory modeling with collaborative design spaces. ACM Transactions on Graphics 28, 5 (2009), 167.

[29] Ye Tao, Guanyun Wang, Caowei Zhang, Nannan Lu, Xiaolian Zhang, Cheng Yao, and Fangtian Ying. 2017. Weavemesh: A low-fidelity and low-cost prototyping approach for 3D models created by flexible assembly. In Proceedings of the $2017 \mathrm{CHI}$ Conference on Human Factors in Computing Systems. 509-518.

[30] Rundong Tian, Sarah Sterman, Ethan Chiou, Jeremy Warner, and Eric Paulos. 2018. Matchsticks: Woodworking through improvisational digital fabrication. In Proceedings of the 2018 CHI Conference on Human Factors in Computing Systems. 1-12.

[31] Michael Wessely, Theophanis Tsandilas, and Wendy E Mackay. 2018. Shape-aware material: Interactive fabrication with shapeme. In Proceedings of the 31st Annual ACM Symposium on User Interface Software and Technology. 127-139.

[32] Karl D.D. Willis, Juncong Lin, Jun Mitani, and Takeo Igarashi. 2010. Spatial sketch: Bridging between movement and fabrication. In Proceedings of the Fourth International Conference on Tangible, Embedded, and Embodied Interaction. 5-12.

[33] Karl D.D. Willis, Cheng Xu, Kuan-Ju Wu, Golan Levin, and Mark D. Gross. 2011. Interactive fabrication: New interfaces for digital fabrication. In Proceedings of the Fifth International Conference on Tangible, Embedded, and Embodied Interaction (Funchal, Portugal) (TEI '11). 69-72.

[34] Chauncey Wilson. 2013. Brainstorming and Beyond: A User-centered Design Method. Newnes.

[35] Robert F Woodbury and Andrew L Burrow. 2006. Whither design space? Ai Edam 20,2 (2006), 63-82.

[36] Xiuming Zhang, Tali Dekel, Tianfan Xue, Andrew Owens, Qiurui He, Jiajun Wu, Stefanie Mueller, and William T. Freeman. 2018. MoSculp: Interactive visualization of shape and time. In Proceedings of the 31st Annual ACM Symposium on User Interface Software and Technology. 275-285.

[37] Clement Zheng, Ellen Yi-Luen Do, and Jim Budd. 2017. Joinery: parametric joint generation for laser cut assemblies. In Proceedings of the 2017 ACM SIGCHI Conference on Creativity and Cognition. 63-74. 